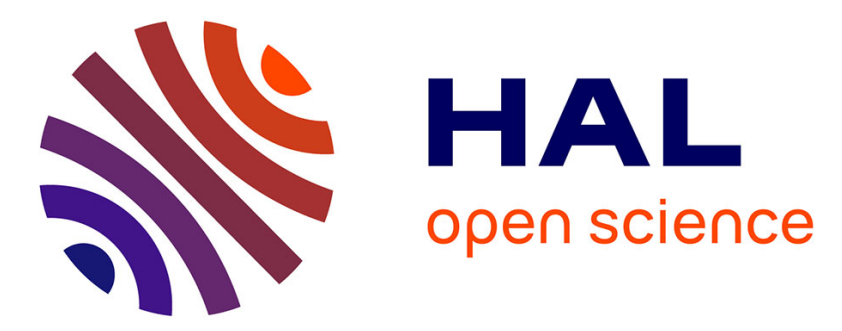

\title{
From 'molecules of life' to new therapeutic approaches, an evolution marked by the advent of artificial intelligence: the cases of chronic pain and neuropathic disorders
}

Jean-Louis Kraus

\section{To cite this version:}

Jean-Louis Kraus. From 'molecules of life' to new therapeutic approaches, an evolution marked by the advent of artificial intelligence: the cases of chronic pain and neuropathic disorders. Drug Discovery Today Biosilico, 2021, 10.1016/j.drudis.2021.01.009 . hal-03142522

\section{HAL Id: hal-03142522 \\ https://hal-amu.archives-ouvertes.fr/hal-03142522}

Submitted on 16 Feb 2021

HAL is a multi-disciplinary open access archive for the deposit and dissemination of scientific research documents, whether they are published or not. The documents may come from teaching and research institutions in France or abroad, or from public or private research centers.
L'archive ouverte pluridisciplinaire HAL, est destinée au dépôt et à la diffusion de documents scientifiques de niveau recherche, publiés ou non, émanant des établissements d'enseignement et de recherche français ou étrangers, des laboratoires publics ou privés.

\section{(1) (1) $\$$}

Distributed under a Creative Commons Attribution - NonCommercial - NoDerivatives 44.0 
Please cite this article in press as: Kraus, J.L. From 'molecules of life' to new therapeutic approaches, an evolution marked by the advent of artificial intelligence: the cases of chronic painand neuropathic disorders, Drug Discov Today (2021), https://doi.org/10.1016/j.drudis.2021.01.009 .Drug Discovery TodayVolume 00, Number 00January 2021

REVIEWS

From 'molecules of life' to new therapeutic approaches, an evolution marked by the advent of artificial intelligence: the cases of chronic pain and neuropathic disorders

Jean-Louis Kraus IBDM-CNRS-Aix-Marseille University, Campus de Luminy, 13288 Marseille Cedex 09, France

The large families of the molecules of life are at the origin of the discovery of new compounds with which to treat disease. The arrival of artificial intelligence (Al) has considerably modified the search for innovative bioactive drugs and their therapeutic applications. Conventional approaches at different organizational research levels have emerged and, thus, Al associated with gene and cell therapies could supplant conventional pharmacotherapy and facilitate the diagnosis of pathologies. Using the examples of chronic pain and neuropathic disorders, which affect a large number of patients, I illustrate here how Al could generate new therapeutic approaches, why some compounds are seen as recreational drugs and others as medicinal drugs, and why, in some countries, psychedelic drugs are considered as potential therapeutic drugs but not in others. Introduction Scientists, social scientists, and humanists must communicate with and understand each other because the survival of human-kind requires the development of scientific programs the social implications and human consequences of which have to be taken into account. Regardless of the applications of their discoveries, scientists in general and medicinal chemists in particular as creators of new molecules, should always recall the wisdom of Indian Iroquois tribes: before taking a decision that could have consequences for the future of the tribe, Iroquois chiefs evoked the impact that this decision would have had on seven past Iroquois generations [1].Today, although new technology inventors think about the impact of their discovery on future human generations, most do not consider the negative impact they might have had on past generations. This statement holds true for the discovery of new molecules regardless of their fields of application, be it health, agriculture, or advanced materials for industrial purposes, among others. Thus, wisdom should be the 
prime quality of chemists creating new molecules, who should never forget that molecules atthe origin of life on Earth are at the base of the current pharmaco-peia used by humans. Small molecules of life at the basis of the world pharmacopeia Starting from organic molecules derived from carbon and other elements, such as hydrogen, oxygen, sulfur, and nitrogen, millions of combinations exist in nature or have been synthesized, including those upon which life itself is based. The most significant are the molecules that constitute the code of life, which have revolutionized our understanding of biological phenomena. Nature has equipped every cell with a complete set of blueprints stored in the nucleus in the form of molecules of DNA, involved in genetics and protein synthesis [2].Then come the so-called 'molecules of mercy', namely pain relievers. The most popular is perhaps acetyl salicylic acid (aspirin), medicinally used to alleviate common aches and pain. Many of the established organic molecule-based pain relievers occur naturally in plants, with morphine, which is 50 times as potent as aspirin, a notable example. Related to the morphine family, numerous structurally related analogs and other drugs, such as heroin, methadone, codeine, pentazocine, cocaine, procaine, atropine, and scopolamine, have been described. Sometimes addictive, some-times sedative, such drugs, including barbiturates and tranquilizers, have their place in medicine if used correctly [3].The fundamental problem related to such drugs is that they are open to abuse. Feel tense? Take a pill. Can't sleep? Take a pill. How many times do we hear such refrains? Humans take energizers, sedatives, analgesics, and even hypnotics to better cope with their pressure-filled lives. Even Cleopatra supposedly took sleeping pills while Mark Anthony was away on a trip! Medicinal chemists have synthesized specific drugs to satisfy increasingly pressing demands from society, resulting in compounds such as barbiturates and tranquilizers (e.g., phenothiazine, meprobamate or chlordiazepoxide). Unfortunately, drug-taking by itself is rarely a cure for psychological conditions, and some of these drugs can be dangerous if used without proper guidance. In addition to drugs for pain and psychological conditions, humans have also exploited naturally occurring compounds to create specific molecules to treat microbial disease. For example, quinine and other antimalarials, sulfanilamide, penicillin, streptomycin, chloramphenicol, and tetracyclines were developed against resistant strains of microorganisms. However, given the prevalence of multidrug-resistant organisms, there is a continuing need to create and synthesize new antibiotics to control such organisms [4].The steroid family and related compounds represent a major breakthrough for medicinal 
chemistry development, because these compounds have various important biological functions, including as vitamins, sex hormones (testosterone, estradiol, and progesterone), contraceptives (norethindrone) and arteriosclerotics (cholesterol), which represent major areas of steroid involvement [5]. Another family of compounds with diverse hormone-like effects are prostaglandins, found in almost every tissue in humans and other animals, and which have a variety of effects, including as vasodilators, in platelet aggregation, inflammation, or smooth muscle contraction [6]Our unending search for life's meaning, deep psychological satisfaction, and insights into immortality have led us to collect, catalog, and even concoct substances that allow us to transcend normal physical limitations and routines. This is why humans have been interested in so-called 'molecules of mysticism': the mind changers [7]. Humans have eaten, imbibed, sniffed, and inject extracts of plant and animal origin to achieve altered consciousness. Four classes of molecule that affect mental processes can be distinguished. Most fit into the classification of hallucinogenic or psychomimetic dugs, or significant mind-altering molecules: (i) lysergic acid derivatives (LSD, alkaloids from ololiuqui, etc.); (ii) phenethylamine compounds (mescaline, amphetamines, etc.); (iii) tryptamine-based molecules (psilocin psilocybin, bufotenine, etc.); and (iv) cannabinoids from Cannabis sativa(hashish, marihuana, etc.)The suggestion that doctors should be paid for keeping people healthy, rather than curing them after they get sick, has been at the origin of the growing interest in vitamins and in so-called 'health foods'. It is also why medicinal chemists have been involved in the creation, synthesis, and biological studies of molecules of growth and health. These molecules are mainly vitamins [8], discovered primarily by scientists seeking to cure specific diseases, such as beriberi, facial tissue deterioration and related forms of dermatitis, pellagra, scurvy, and night blindness, which all arise from vitamin deficiencies, mainly vitamin B1 (thiamine), vitamin B2 (ribofla-vin), vitamin B6 (pyridoxine), nicotinic acid (niacin) and nicotinamide, vitamin C (ascorbic acid), vitamin A (retinol), and vitamin E (tocopherols). The structure and chemistry of these molecules of growth and health differ substantially from each other, but have important role as cofactors in regulating appropriate enzyme actions. Who among us cannot recall the effect of head colds or simply blocked nasal passages on our sense of taste? Thus, molecules of the senses (taste, odor, and attraction) have been isolated and synthesized by chemists: (i) taste molecules: monosaccharides(glucose and related analogs), disaccharides (maltose and lactose),amino acids and peptides that have a sweet taste.; (ii) odor molecules, which 
can belong to different types of fragrance: camphoraceous, (D-camphor); pungent, (formaldehyde, acetic acid, allyl alcohol); ethereal, (chloroform, propyl alcohol), floral, (geraniol,terpineol, ionones, benzyl acetate); peppermint (menthone, cyclohexanone, piperitol), musky (cyclohexadecanone, androstanol, muscone); and putrid (cadaverine, skatole, amylmercaptan, hydrogen sulfide); and (iii) molecules of attraction(pheromones), which are chemical substances that allow intraspecies communication. The structure and chemistry attraction pheromones, such as disparlure and propylure, differ substantially from each other depending on the species. The common denominator among these molecules is that they are volatile, long-lasting, and have a potential irritating quality. Terpenoids and related molecules are common types of molecule isolated from insects andants and which are used in defense, such as citronellal, geraniol, citral, farnesal, limonene, and perillene. Benzoquinones and related analogs are among the most widely distributed arthropod defensive compounds. Other compounds, such as cresols, salicylaldehyde, benzoic acids, and benzaldehyde, have also been iso-lated. Synthetic organic chemists have focused on insect pheromones with the aim of controlling the destructive feature sof insect populations. Numerous attractants and repellents have been discovered, such as trimediure against Mediterranean fruit flies, methyl benzoate against mites, N-butylacetanilide against ticks, and 2-butyl-2-ethyl-1,3-propane diol against mosquitos. Although such compounds were designed to be effective, safe, and specific for the particular species, some are ineffective and can even be toxic.Given the earlier discussion of our exploitation of naturally occurring molecules, the resulting large numbers of approved pharmaceutical medicines worldwide, and the fact that their effects are sometimes suboptimal, would it now be better to search for new health treatments using gene or cellular therapies rather than to continue the research and development of small molecules? Most traditional drugs, as well as $>90 \%$ of therapeutics current-ly marketed, are small molecules [9]. The key drawbacks of conventional small-molecule therapeutics are that they can do only one thing and that they keep doing it, regardless of the physio-logical state of the patient, because they are not equipped to receive feed-back from the body. One significant example are beta-blocker drugs, which keep blocking the functioning of the heart regardless of the physiological state of the patient [10]. One area where small molecules are, and will likely remain, the best possible therapeutic tools, is the fight against bacteria, fungi, and viruses. Small-molecule inhibitors are effective antimicrobials because they target enzymes specific to the target 
species. However, when our own proteins need to be regulated for therapeutic purposes, the 'single-mindedness' of enzyme inhibitors or receptor ligands, as well as their unresponsiveness to signals sent by the body, remain problematic. This is why new immunological or genomic therapies are emerging and beginning to replace existing standard small-molecule drugs.

New therapeutic cellular approaches: the use of large molecules Cellular behaviors, such as adhesion, migration, and cell death by apoptosis or other mechanisms, are mediated and regulated via protein-protein interactions (PPIs). The ability to selectively disrupt or enhance individual PPIs gives unprecedented leverage over the cell, essentially telling the cell what to do, and when to do it, in a language that it understands [11]. This could help to elucidate the intricacies of cell signaling, which is currently a significant challenge in biological research. By introducing a protein with modified signaling properties into a cell, it is possible to affect the behavior of that cell. Cancer cells and dying neurons represent obvious targets. Given that the biological function of signaling proteins is to deliver chemical messages, if one could tell cancer cells to stop proliferating and dying neurons to stay alive, crucial disease could be cured or prevented. Viral and non viral gene delivery systems, as well as the identification of promoters driving expression in specific cell types, are under development [12]. Arecent application of such a technique was the treatment of Leber congenital amaurosis (LCA), a rare inherited eye disease that appears at birth or during the first few months of life; using adeno-associated virus gene therapy, it was possible to restore vision in patients with LCA [13].For therapeutic purposes, it is often necessary to selectively enhance or reduce only one interaction with a particular signaling protein, out of several that are normally engaged in the target reaction. Arrestins, a small family of proteins, important for regulating signal transduction at G-protein-coupled receptors, repre-sent a significant model for specific interaction approaches, because these elongated molecules have a variety of roles in several biological processes, such as the regulation and localization of phosphodiesterase, programmed cell death, infectious diseases, host-pathogen interactions, regulation of GTPases, airway epithelium and asthma, as well as pain and anesthesia [14]. Arrestin binding to a receptor blocks further $\mathrm{G}$ proteinmediated signaling, targets receptors for internalization, and redirects signaling to alternative $G$ protein-independent pathways [15].

Drugs for medical use: the therapeutic context 
The search continues for new drugs or molecules with improvedbiological properties, which ideally would only have beneficialhealth benefits. To address this challenge, several questions arise,such as: (i) in what therapeutic context can drugs be used and inwhat form? (ii) How can we measure the benefit-risk ratio of themedical use of drugs? (iii) How can we supervise the productionand sale of drugs for medical use? (iv) What is the differencebetween a drug and a medicinal drug? (v) Why are certain mole-cules for recreational use struck by pharmacological infamy? Let usconsider first the case of psychoactive drugs. Thirty countries have legalized cannabis for therapeutic use. France remains resistant to the commercialization of treatmentsinvolving cannabinoid derivatives for certain forms of epilepsy,multiple sclerosis, or chronic pain. However, behind the possibleuse of cannabinoids, there is a whole family of molecules that arebeginning to be researched and used for such conditions, the so-called 'molecules of mysticism' or 'mind changers' [16].Can we legalize those drugs for medical use? Questions abouttheir use, the lack of legislation, and reluctance around theirtherapeutic application, too often considered as recreational, arestill questions that need to be addressed, because some of thesecompounds could be helpful for patients with chronic pain,spasms (notably linked to multiple sclerosis), or certain childhoodepilepsies. Hallucinogens and psychedelic drugs are being investigated todiscover possible new therapeutic applications. Researchers arestudying the action of hallucinogenics on certain treatment-resis-tant pathologies, such as chronic depression, alcohol and drugdependence, post-traumatic stress disorder, and obsessive-compul-sive disorder (OCD). In France and other countries, some psychedel-ic drugs are illegal, such as ketamine, LSD, MDMA (ecstasy),psilocybin mushrooms, ayahuasca, and ibogaine [17]. Such com-pounds, similar to chemotherapeutic drugs for cancer, or morphinefor pain, would need to be administrated cautiously. Although suchpsychedelic drugs are listed as illegal because they alter states ofconsciousness, they could be also interesting as medical treatments.As an example, A. Huxley took mescaline, the active alkaloid ofpeyote, the Indian cactus, resulting in colorful visions accompaniedby various psychological phenomena [18]. However, the use ofhallucinogens and narcotics as 'antiintellectual' drugs would bean inappropriate way to find answers for medical conditions currently lacking effective treatments $[19,20]$.A recent study reported that psilocybin was effective at reducingsymptoms in humans with treatment-resistant depression [21].Psilocybin can help patients become more optimistic and could bean effective way to 
treat tobacco [22] and alcohol abuse [23].Patients who used psychedelics have lower rates of psychologicaldistress and suicidality compared with those who do not usepsychedelics; for example, psilocybin made patients living withlung cancer more comfortable compared with control groups [20]. Even if results from clinical trials with psilocybin were promising,psychotherapy trials confirming its efficacy and safety are stillneeded.

Nevertheless, the potential effects of psilocybin on de-pression, anxiety, and palliative care should be considered infuture studies.

The case of drugs for chronic pain

Research is beginning to focus on searching for new syntheticmolecules and new immunopharmacological approaches to re-place conventional drug therapies. The development of therapiesfor chronic pain is an example of research duality between newsynthetic molecules (small molecules) and immunologicalapproaches involving large molecules (proteins, chemokines, and neurokines). Chronic pain is a major problem for humanhealth, affecting $20 \%$ of the world's population; it also has aconsiderable economic impact and its impact will continue toincrease with increasing life expectancy; in addition, chronic painis the number one cause of long-term disability in the USA; $27 \%$ ofchronic pain is related to low back pain; $77 \%$ of patients withchronic pain report feeling depressed because of their condition; and $66 \%$ of patients seek treatment from medical doctors, $25 \%$ from chiropractors, and $15 \%$ from pain specialists [. Opioids continue to be prescribed to patients with chronic paindespite the fact that their use is broadly accepted to be mosteffective for acute pain, cancer pain, and palliative care. Addition-ally, only $23 \%$ of patients with chronic pain found opioids to beeffective [24]. Chronic and neuropathic pain treatments mainlyrely on chemotherapeutic agents. Chronic (nociceptive) pain fromtissue inflammation or damage (as in rheumatoid arthritis andcancer pain) is best treated with opioids, such as opiorphin [25], whereas neuropathic pain (caused by a damaged or dysfunctionalnervous system) is treated more effectively by drugs such as tricy-clic antidepressants [26], serotoninnorepinephrine reuptake inhi-bitors, and anticonvulsants $[27,28]$. For example, lacosamide [a USFood and Drug Administration (FDA)-approved drug] abolishesthe disease-specific phenotype by blocking induced pluripotentstem cell (iPSC)-derived nociceptors [29]. Unfortunately, the use ofmost standard chemical synthetic drugs, including paracetamol-like drugs, acetyl salicylic analogs, noramidopyrine, floctafenin, nefopam, codeine derivatives, and other potent antalgic drugs, including morphine-related analogs, often induce adverse 
effects; thus, new immunological treatment approaches are under inves-tigation.One such approach reported was the injection of a chemokine-like protein TAFA4, in animal models of inflammatory or nerveinjury-induced pain. TAFA4, a chemokine-like protein, was shownto modulate injury-induced mechanical and chemical pain hyper-sensitivity in mice [30]. Another approach was based on thedevelopment of molecular-specific sodium channel (NAV 1.7,NAV 1.8) blockers [31,32]. Natural toxins, such as saxitoxin [33]and aryl sulfonamide [34], show promise for treatment of neuro-pathic pain with minimal adverse effects.

Role of Al in the development of new drugs anddiagnostics for chronic pain and neuropathic disorders The use of $\mathrm{Al}$ in the health sector research has considerablymodified conventional approaches at different levels in the drugdiscovery process.Design, synthesis, identification, and screening of newtherapeutic moleculesThe synthesis of molecules remains one of the most importantchallenges for organic and medicinal chemists. Performing algo-rithms, such as the Chematica Computer Program [35], has shownthe effectiveness of Al in providing chemists with realistic solu-tions for molecule synthesis. For several decades, software allow-ing predictive structural drug and protein determination havebeen available, such as predictive nuclear magnetic resonance(NMR), and mass spectrometry (MS)/liquid chromatography(LC) software [36]. Some software provides support for developingmolecular models de novo, whereas others directly support aspectsrelated to constructing molecular models, including moleculargraphics, interactive drawing and conformational editing. Suchsoftware is now frequently used by medicinal chemists and struc-tural biologists [37]. Using Al techniques with robot algorithmsenables researchers to quickly conduct millions of chemical, ge-netic, and pharmacological tests to rapidly identify active com-pounds, antibodies, or genes that modulate a particular pathway;such highthroughput screening is increasingly the technology ofchoice in drug discovery and is relevant to both the biology andchemistry fields [38].

Al in medicinal biology, diagnostic assistance programs, andpersonalized patient treatment programsThe 'omics revolution requiring Big Data mining has been themajor driving force behind the development of Al. The firstcomprehensive systems-biology dynamical model explaining pat-terning in planarian regeneration provided an automated, highlygeneralizable framework for identifying the underlying controlmechanisms responsible for the dynamic 
regulation of growth andform [39]. Such systems could be helpful for clinical research inregenerative medicine.The first aim of health-related Al applications is to analyzerelationships between prevention or treatment techniques andpatient outcomes. Various specialties have shown increased use ofAl to this end, such as disease diagnosis using computed tomogra-phy (CT), and magnetic resonance imaging (MRI) techniques [40]; deep learning algorithms have been developed to search reportsand detect patterns that imply drug-drug interactions [41]; skincancer was detected more accurately using an Al system that used adeep learning convolutional neural network compared withdermatologist-based assessments [42]; and in radiology, an algo-rithm was created that could better detect pneumonia in patients $[43,44]$.The ability to monitor patients using Al could allow for directcommunication to physicians in terms of whether possible diseaseactivity might have occurred [45]. In addition, an Al tool that scanselectronic health record (her) data can accurately predict thecourse of disease in a person [46]. The primary aim of health-related $\mathrm{Al}$ applications is to analyze relationships between preven-tion or treatment techniques and patient outcomes. Among theseapplications, personalized medicine, and patient monitoring andcare represent the major challenges to be addressed [47]. Al appli-cation for the development of treatment protocols, such astreatmentbiomarker combinations in colon cancer (Erbitux-EFGR) and lung cancer (Xalkori-ALK), good examples of person-alized treatments [48]. Other successful example of Al applicationis the prediction of correct personalized dosing treatments.Al applications in chronic painTechnologies to accurately evaluate a patient's pain level arenecessary given that half of patients with chronic pain also expe-rience anxiety or depression at some point because of their pain.Difficulties arise when carers try to ascertain a patient's pain levels, because sensations are inherently hard for patients to express.However, different Al options are available for this purpose:The My Intelligent Assistant (MIA) software was developed by aFrench start-up Sante' Net (www.mia-software.com) incorporatedin a bot. It regularly asks patients with chronic pain questionsabout their pain intensity, fatigue, and how frequently they aretaking medication. This allows patients to spontaneously notepain as it occurs, relaying the information to their carer andkeeping them informed. It also allows medical professionals toclearly identify when a patient needs to take a sleeping pill. Thisalso helps to combat the over-reliance on powerful and highlyaddictive analgesics, such as morphine. This algorithm also allows 
Patients to manage their pain autonomously with personalizedtherapeutic advice based on the data that they provide.Al can be used to assess pain levels through facial recognitiontechnology, which notes movements such as furrowed eyebrows, squeezed lips, and raised cheeks, to calculate pain levels. Thistechnology can also be used by anesthetists when determiningthe dose of morphine required for a patient. However, suchtechnology is only used for treating sharp, short-lasting pain.For patients who endure pain for a long time, the body alarmsystem stops working in a way which cannot be monitored by suchtechnology.Al can also be applied to neuropathic pain measurement basedon brain imaging. For example, brain images from individualpatients with chronic low back pain were collected when theirpain was at a low (baseline) state. The patients then performedphysical maneuvers intended to temporarily increase their pain,including movements such as sit-ups and back-arching motions, and then underwent repeated imaging. By using a machine-learning $(\mathrm{ML})$ approach to compare brain images from individualpatients in the two states, researchers identified other specificbrain areas that were activated by exacerbated pain; by contrast,in normal pain, brain activity was increased only in the thalamus,a sensoryprocessing station in the brain [49].Al can also be exploited as an imaging biomarker for clinicalpain measurement. A specific biomarker that could be used fordiagnostic purposes to prove whether someone has chronic painwould be of value [50]. Although in the early stages of develop-ment, methods including structural and functional MRI andelectroencephalography, are being used. These methodologieshave already produced encouraging predictive models that havebeen tested on clinical populations [51].

Al applications in neuropathic disorders

Disruptive technologies, such as ML algorithms, are especiallyrelevant to the discovery of compounds targeting neuropsychi-atric disorders, in which current therapies lead to many off-targeteffects. Leveraging Al technologies, new molecules are beingderived from psychoactive small-molecule compounds (SMC), such as psilocybin or LSD, to tackle unmet medical needs inmental healthcare. The development of sophisticated ML algo-rithms provides a set of tools that can improve discovery anddecision-making for specific questions based on sufficient, high-quality data. A typical application of SMC technology is psylo-cibin therapy [19], which aims to develop compounds with thetherapeutic benefits of psilocybin for depression, but with im-proved characteristics, such as quicker drug release and absorption or the absence of hallucinogenic effects typical topsychedelics. These technologies do 
not aim to replace classicalpsychedelics completely. Instead, the reformulation of psychoactive seed SMCs endeavors to make psychedelic therapy moreaccessible to a larger proportion of the population, such as thosewho react unfavorably to the hallucinatory effects or thoseindividuals with cardiac problems .Concluding remarks

'Nature invents and man tries to copy it'. From the formation andevolution of initial molecules that gave rise to families of increas-ingly complex natural molecules with extraordinary biologicalproperties, organic chemists in general and medicinal chemistsin particular, have never stopped trying to create molecular struc-tures with improved biological properties for use in the clinic. Toachieve this, research chemists have teamed up with biologists,doctors, physicists, and mathematicians to respond as effectivelyas possible to the major challenges of keeping humans healthy.Technologies offered by Al have been used at all levels of research,from the development of new methods of synthesis to methods ofapplication leading to personalized medicine. Although suchapproaches are likely to lead to exciting developments in humanhealthcare, they are likely to come at a cost, and it is currentlyunclear whether all those who would benefit from them will beable to afford to do so.

Declaration of Competing Interest The authors declare that they have no known competing financialinterests or personal relationships that could have appeared toinfluence the work reported in this paper.

Acknowledgments

Financial support is acknowledged from the Institut de Biologie duDé veloppement de Marseille (IBDM), Aix-Marseille-University andCNRS-UMR 7288. Drs Le Bivic and Kodjabachian are acknowledged for research facilities offered.

\section{References}

1 Dixon-Kennedy, M. (1998) Native American Myth \& Legend. Brockhampton Press

2 Watson, J.D. (1970) Molecular Biology of the Gene. W.A. Benjamin

3 Central Intelligence Agency (2018) The CIA World Fact Book 2018-2019.

SkyhorsePublishing

4 Morris, A.M. et al. (2019) Research needs in antibiotic stewardship. Infect. ControlHospital Epidemiol. 40, 1334-13435 
Lednicer, D. (2011) Steroid Chemistry at a Glance. A Concise History of the Study ofSteroids. Wiley

6 Ricciotti, E. and Fitzerald, G. (2011) Prostaglandins and Inflammation. Arterio.Thromb. Vasc. Biol. 319861000

7 Goodman, M. and Morehouse, F. (1973) Organic Molecules in Action. Gordon \&Breach Publishing Group

8 Combs, G.F. (2007) The Vitamins. Elsevier

9 Hopkins, A.L. and Gromm, C.R. (2002) The druggable genome. Nat Rev Drug Discov.1, 727730

10 Frishman, W.H. et al. (2005) Current Cardiovascular Drugs. Current Science Group 11 Guervich, E.V. and Guervich, V.V. (2014) Therapeutic potential small moleculesand engineered proteins. Handb. Exp. Pharmacol. 219, 1-12

12 Bartel, M.A. et al. (2012) Directed evolution of novel adeno-associated viruses fortherapeutic gene delivery. Gene Ther. 19, 694-700

13 Simonelli, F. et al. (2010) Gene therapy for Leber's congenital amaurosis is safe andeffective through 1.5 years after vector administration. Mol. Ther. 18, 643-650 14 Gurevich, V.V. and Guervich, E.V. (2006) The structural basis of arrestinmediatedregulation of G-protein-coupled receptors. Pharmacol. Ther. 110, 465-502 15 Smith, J.S. et al. (2018) Biased signalling: from simple switches to allostericmicroprocessors. Nat. Rev. Drug Discov. 17, 243-260 16 National Academies of Sciences, Engineering, and Medicine (2017) The HealthEffects of Cannabis and Cannabinoids: The Current State of Evidence andRecommendations for Research. The National Academies Press

17 Doblin, R.E. et al. (2019) The past and future of psychedelic science. J. PsychoactiveDrugs $51,93-97$

18 Huxley, A. (1954) The Doors of Perception. Chatto \& Windus 19 Griffiths, R.R. et al. (2018) Psilocybin-occasioned mystical-type experience incombination with meditation and other spiritual practices produces enduringpositive changes in psychological functioning and in trait measures of prosocialattitudes and behaviors. J. Psychopharmacol. 32, 49-69 
20 Griffitths, R.R. et al. (2016) Psilocybin produces substantial and sustained decreasesin depression and anxiety in patients with life-threatening cancer: a randomizeddouble-blind trial. J. Psychopharmacol. 30, 1181-1197

21 Carhart-Harris, R.L. et al. (2017) Psilocybin for treatment-resistant depression: fMRImeasured brain mechanisms. Sci. Rep. 7, 13187

22 Johnson, M.W. et al. (2017) Long-term follow-up of Psilocybin-facilitated smokingcessation. Am. J. Drug Alcohol Abuse 43, 127

23 Bogenschutz, M.P. et al. (2015) Psilocybin-assisted treatment for alcoholdependence: a proof of concept study. J. Psychopharmacol. 29, 289-299

24 Dalhamer, J. et al. (2018) Prevalence of chronic pain and high-impact chronic painamong adults-United States. MMWR Mortal. Wkly. Rep. 67, 1006

25 Rougeot, C. et al. (2010) Systemically active human opiorphin is a potent yet nonaddictive analgesic without drug tolerance effects. J. Physiol. Pharmacol. 61, 483-490 26 Gilman, P.K. (2007) Tricyclic antidepressant pharmacology and therapeutic druginteractions updated. Br. J. Pharmacol. 151, 737-748

27 Wright, M.E. and Rizzolo, D. (2017) An update on the pharmacologic managementand treatment of neuropathic pain. JAAPA 30, 13-17

28 Cashman, J.R. and Ghirmai, S. (2009) Inhibition of serotonin and norepinephrinereuptake and inhibition of phosphodiesterase by multi-target inhibitors as potentialagents for depression. Bioorg. Med. Chem. 17, 6890-6897

29 Namer, B. et al. (2019) Pain relief in a neuropathy patient by lacosamide: proof ofprinciple of clinical translation from patient-specific iPS cell-derived nociceptors.EBioMedicine 39, 401-408

30 Delfini, M.-C. et al. (2013) TAFA4, a chemokine-like protein, modulates injury-induced mechanical and chemical pain hypersensitivity in mice. Cell Rep. 5, 378 31 Hameed, S. (2019) Nav1.7 and Nav1.8: role in the pathophysiology of pain. Mol. Pain15 1744806919858801

$32 \mathrm{Siu}, \mathrm{R}$. et al. (2019) Voltage gated sodium channels as therapeutic targets for chronicpain. J. Pain Res. 1, 2709-2722

33 Cusick, K.D. and Sayler, G.S. (2013) An overview on the marine neurotoxin,saxitoxin: genetics, molecular targets, methods of detection and ecologicalfunctions. Mar. Drugs 11, 991-1018 
$34 \mathrm{Kim}$, J.H. et al. (2016) Synthesis and T-type calcium channel-blocking effects of aryl(1,5disubstituted-pyrazol-3-yl) methyl sulfonamides for neuropathic paintreatment. Eur. J. Med. Chem. 123, 665-672

35 Kluczick, T. et al. (2018) Efficient synthesis of diverse medicinally relevant targetsplanned by computer and executed in the laboratory. Chem 4, 522-532

36 Berg, J.M. et al. (2002) Three-dimensional protein structure can be determined byNMR spectroscopy and X-ray crystallography. In Biochemistry (5th edn) (Berg, J.M.,ed.), pp. XXXYYY, W.H. Freeman

37 Liao, C. et al. (2011) Soft-ware and resources for computational medicinalchemistry. Future Med. Chem. 3, 1051085

38 Macarron, R. et al. (2011) Impact of high-throughput screening in biomedicalresearch. Nat. Rev. Drug Discov. 10, 188-195

39 Lobo, D. and Levin, M. (2015) Inferring regulatory networks from experimentalmorphological phenotypes: a computational method reverse-engineers planarianregeneration. PLoS Comput. Biol. 11, e100429

40 Zhang, M.-J. et al. (2015) Analysis on value of CT and MRI clinical application indiagnosis of middle-aged patients with multiple cerebral infarction. Int. J. Clin. Exp.Med. 8, 1712317127

$41 \mathrm{Xu}, \mathrm{B}$. et al. (2019) Incorporating user generated content for drug interactionextraction based on full attention mechanism. IEEE Trans. NanoBioscience 18, 360-367 42 Haenssle, H.A. et al. (2018) Man against machine: diagnostic performance of a deeplearning convolutional neural network for dermoscopic melanoma recognition incomparison to 58 dermatologists. Ann. Oncol. 29, 1836-1842

43 Rajpurkar, P. et al. (2017) CheXNet: radiologist-level pneumonia detection on chestX-rays with deep learning. arXiv 2017 arXiv:1711.05225

44 Bahl, M. et al. (2018) High-risk breast lesions: a machine learning model topredict pathologic upgrade and reduce unnecessary surgical excision. Radiology286, 810-818 45 Wahl, B. et al. (2018) Artificial intelligence (Al) and global health: how can Alcontribute to health in resource-poor settings? BMJ Global Health 3, e000798 46 Hetch, J. (2019) Fixing a broken record, the future of electronic health records. Nature 573, S114-S116 
47 Lu, Y.F. et al. (2014) Personalized medicine and human genetic diversity. Cold Spring Harb. Perspect. Med. 4 (9), a008581

48 Vincent, M.D. et al. (2012) Biomarkers that currently affect clinical practice: EGFR,ALK, MET, KRAS. Curr. Oncol. 19 (Suppl. 1), S33-S44

49 Lötsch, J. and Ultsch, A. (2018) Machine learning in pain research. J. Pain 159,623-630

50 Long, X. et al. (2016) Sustained effects of acupuncture stimulation investigated withcentrality mapping analysis. Front Hum. Neurosci. 18, 510

51 Van der Miesen, M.M. et al. (2019) Neuroimaging-based biomarkers for pain: State of the fielding current directions. Pain Rep. 4 (4), 